If the authors were to repeat this quality improvement project, it would be useful to assess the implication of poor handover on infant care. For example missed/ late blood samples or delayed discharge.

Message for others The message conveyed by this project is that every ward should have an effective handover and that with using simple measures dramatic differences can be made. This project identified a lack of effective handover on a postnatal ward which led to delayed or missed patient care. Using a handover tool designed following staff feedback, handover between staff members improved significantly and this positively impacted on patient care.

Effective handover helps ensure patients receive appropriate and timely care. Potentially serious results are not forgotten about or missed when staff finish duty. It is essential as clinicians to ensure patients receive the best care and effective handover is an integral part of this.

\section{G584(P) SBAR HANDOVER TOOL: IMPROVING MATERNITY - PAEDIATRIC COMMUNICATION AT DELIVERIES}

${ }^{1}$ E Sloper, ${ }^{1} \mathrm{C}$ Edmonds, ${ }^{2} \mathrm{H}$ Bailey, ${ }^{2} \mathrm{~L}$ Lewis, ${ }^{2} \mathrm{R}$ Charlton. ${ }^{1}$ Paediatrics, Royal United Hospital Bath, Bath, UK; ${ }^{2}$ Midwifery, Royal United Hospital Bath, Bath, UK

\subsection{6/archdischild-2015-308599.533}

Context The project involves deliveries in our district general hospital delivery unit where a member of the paediatric team is requested to attend. The staff involved include midwives, neonatal nurses, paediatricians and obstetricians.

Problem Following the Root Cause Analysis (RCA) of a neonatal death at our hospital, it was recommended that improvements be made to delivery room handover between midwives and attending paediatricians. Currently handovers follow no specific pattern allowing for important information to be missed. As a result, the appropriate grade of paediatrician and correct equipment may not be present and paediatric staff may be ill-equipped to cope with the impending delivery.

Assessment of problem and analysis of its causes The RCA indicated that the condition of the baby at birth may have been predicted had the paediatrician been given a more detailed handover. A detailed handover may also have prompted them to call for senior support prior to delivery. Further discussion with paediatricians and midwives identified a lack of structure to these handovers, leaving babies at risk. Junior staff also felt that in stressful situations, prompts would ensure important information is remembered. Additionally, parents reported they would be reassured to observe a formal handover between teams.

In other high-risk transfer areas, teams complete checklists to ensure they have the necessary information, staff and equipment to minimise risk. Currently, we have no such checklist for a paediatrician entering a delivery room. Handover relies on informal discussion, often whilst staff perform other tasks. It was therefore felt that a checklist would be a useful aide in prompting a concise, complete handover.

Intervention A search was conducted to find checklists for handover between midwifery and paediatric teams in other units but none could be identified. A checklist has therefore been designed by paediatricians and approved for this study. It covers the information necessary to provide safe initial care to a baby. The checklist has been attached to every resuscitaire in the delivery unit so it is easily accessible.

Study design The checklist was introduced to midwives and paediatricians through briefings at their daily handovers. The checklist was tested on a single day and tests of change made using a Plan, Do, Study, Act (PDSA) cycle. Following further feedback from all staff groups after 2 and 4 weeks, the checklist will be amended again.

Strategy for change The change has been implemented following briefings for staff and a trial of the checklist's use. It has been amended according to initial feedback and will be attached to all resuscitaires. Further feedback will be obtained at 2 and 4 weeks after formal introduction of the checklists and they will be updated accordingly. Once formalised, the checklists will be incorporated into midwifery teaching sessions and doctor's departmental induction programmes.

Measurement of improvement Analysis will be qualitative and will focus on staff satisfaction and suggested points for improvement. Parent satisfaction will be assessed via patient surveys. Quantitative assessment of delivery outcomes cannot be undertaken within this short timescale because the number of significant neonatal resuscitations is low.

Effects of changes It is hoped that there will be improved communication in the delivery unit resulting in better anticipation and safer care, fewer crash calls for middle grade paediatricians, higher levels of staff confidence and improved parent confidence in the staff caring for their baby.

Lessons learnt We hope to learn how to devise a safe means of communicating essential information between teams.

Message for others Safe handover is a GMC requirement for good practice and we have identified a high-risk area where there are no clear guidelines for this process. Implementing our checklist has allowed the process to be formalised, making delivery room handover safer, quicker and less liable to error.

\section{G585(P) TRANSITION FROM CHILDREN'S TO ADULT DIABETES CLINIC -PATIENT'S VIEWS}

C Patankar, P Williams. Paediatrics, Medway Maritime Hospital, Gillingham, UK

10.1136/archdischild-2015-308599.534

Context The best method of providing transition of young people with diabetes is a complex and much debated subject.

Problem There is a dearth of empirical evidence on the best approach to the transition process. Involving patients is an integral part of designing and delivering this service

Intervention To obtain input from young people with diabetes regarding the current transition service in the Hospital and also invite suggestions for improvement.

Study design Young people over 16 years and adults up to age of 25 years were included. We distributed survey questionnaires via the paediatrics diabetic clinics, and also posted them to patients who were in an adult service.

Measurement of improvement Responses from the patients.

Effects of changes Results There were 200 patients between the age of 16 yrs and 25 yrs available, with 68 responses. The current system (one joint appointment with the paediatrician and the adult physician, then straight to a young adult clinic) was popular (46.3\%). Most (44.1\%) would like to stay for 1 year in the clinic but few longer than that. A significant number (71.2\%) want the adult doctor to be present in the clinic. Interestingly few (3\%) were interested in a psychologist being present. This may reflect a lack of understanding of the difficulties in the transition process by the patients. It may also reflect the lack of knowledge of how a psychologist can support them. The best time to hold the clinic seems to be at routine clinic 
times. Most would like the appointment to last between 20-30 min. A diabetic nurse run clinic was preferred by $48.4 \%(51.6 \%$ did not want it). The best age to be transferred was between 1619 yrs.

Lessons learnt Patient input and experience can give useful insight when designing a model for transition for young people with diabetes.

Message for others Patient views are imperative to bring about a change in the service made for them.

\section{G586(P) PHOTOTHERAPY MANAGEMENT IN JAUNDICED BABIES: JAUNDICE MANAGEMENT TOOL}

JL McDermott. The Neonatal Intensive Care Unit, Queen Alexandra Hospital, Portsmouth, UK

\subsection{6/archdischild-2015-308599.535}

Context This quality improvement work aimed to improve the management of infants on the postnatal ward (PNW) at Queen Alexandra Hospital, Portsmouth who were undergoing phototherapy for the management of neonatal jaundice. The main staff groups targeted were those involved in the care of infants on the PNW (neonatal trainees, ANNPs, midwives and health care support workers).

Problem In 2010, the National institute for Health and Clinical Excellence (NICE) published evidence-based guidelines aimed at standardising the management of neonatal jaundice across the UK. In 2014, an audit of the management of jaundiced infants on the PNW against NICE standards revealed deficiencies relating to the timing of repeat serum bilirubin measurement (SBR) with only $21 \%$ having a repeat SBR which complied with the NICE standard. This in turn was potentially putting babies at risk from jaundice.

Assessment of problem and analysis of its causes The NICE phototherapy pathway was displayed on a wall on the PNW. Its complex nature meant that many members of staff did not use it to guide their management of infants under phototherapy. Midwives would often ask the neonatal trainees for advice regarding management and timings of future SBR samples. With trainees changing frequently and a variety of level of experience, there appeared to be a lack of consistency in the management of neonatal jaundice.

Intervention A Jaundice Management Tool (JMT) based on the NICE guidance was designed to use alongside the NICE jaundice graphs (see figure 1). This formally documents times of SBRs, guides the phototherapy management, and advises on timings of subsequent SBRs.

Study design A reaudit following a month of using the JMT will investigate whether adherence to NICE guidelines is improved.

Strategy for change The JMT was printed, attached to the jaundice treatment threshold graphs and distributed to all the PNW. At the same time, midwives, healthcare support workers and neonatal trainees underwent training in the use of the JMT.

Measurement of improvement Following a month of using the JMT on the postnatal ward, a formal reaudit will be underway, along with a questionnaire to provide feedback regarding the JMT, and assess how user-friendly it is.

Effects of changes So far the JMT has resulted in improved clinical care with more babies having appropriately timed repeat SBRs. In addition, it appears that the JMT has removed confusion around the timing of repeat SBRs and has encouraged a standardised approach across disciplines who are involved in the care of postnatal ward babies.

Lessons learnt I have learnt that in order to implement the JMT, it was crucial that those involved were engaged in the process and keen to be part of the change in practice. It necessitated me liaising with a multidisciplinary group and I learnt to change my style depending on the disciplines I was addressing.

Message for others In line with several studies relating to practice change, passive dissemination of guidelines is rarely effective. It is not sufficient to merely display guidance but necessitates meeting with the various disciplines and educating them on the need for change and how it will improve patient care

The active dissemination strategy using a multifaceted used in this project seemed effective in improving practice. Useful elements included tailoring the implementation of change to the

\begin{tabular}{|c|c|}
\hline $\begin{array}{l}\text { Name } \\
\text { Date of Birth } \\
\text { Q number } \\
\text { (attach addressograph if available) }\end{array}$ & $\begin{array}{l}\text { If a baby looks ja undiced, perform an SBR or trans cutaneous bilirubin measurement. If result plots } \\
\text { above treatment line, start phototherapy and see below: } \\
\text { 1. Start multiple phototherapy if the SBR is rising rapidly (>8.5micromol/ } / / \mathrm{hr} \text { ) or within } 5 \text { boxes } \\
\text { below the threshold for exchange transfusion after } 72 \text { hours - INFORM CON SULTANT INVOLIVED } \\
\text { 2. Perform FBC, blood group and DAT in all babies treated with phototherapy (heel prick) } \\
\text { 3. Consider other investigations e.g. septic screen investigations depending on the clinical situation } \\
\text { - START Photother apy } \rightarrow \text { Repeat SBR in 4-6 hours }\end{array}$ \\
\hline & - STABLE / FALLING SBR $\rightarrow$ Repea SBR every $8 \mathrm{hrs}$ \\
\hline Time of birth & $\begin{array}{l}\text { - STOP phototherapy if SBR greater than or equal to } 5 \text { boxes below tr eatment line } \\
\text { - CHECK SBR } 12 \text { hours after turning off lights (rebound SBR) }\end{array}$ \\
\hline
\end{tabular}

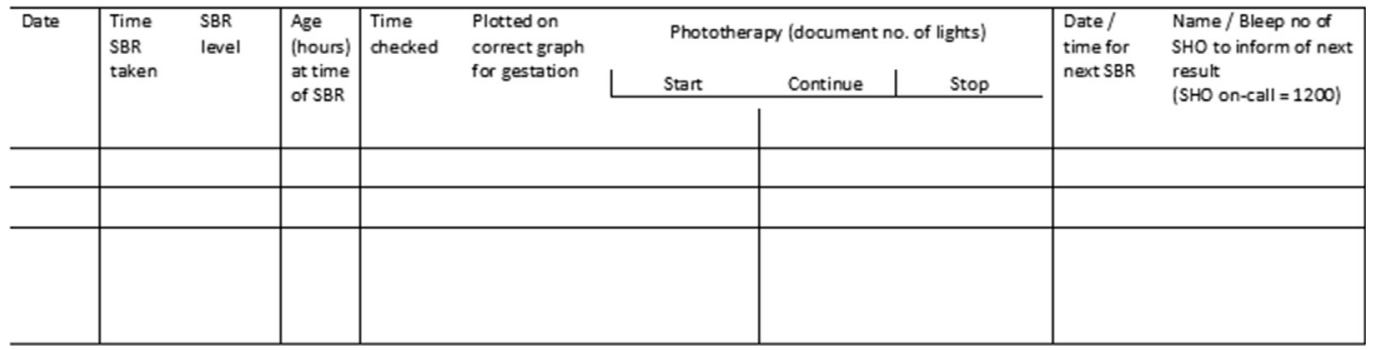

